4486

Assessing the Validity of an ICD-9 and ICD-10 Coding Algorithm for Identifying Cervical Premalignant Lesions Using Administrative Claims Data

Jaimie Zhi Shing, $\mathrm{MPH}^{1}$, Marie Griffin ${ }^{1}$, James C Slaughter ${ }^{1}$, Manideepthi Pemmaraju ${ }^{1}$, Edward F Mitchel ${ }^{1}$, Rachel S Chang ${ }^{2}$, and Pamela C Hull

${ }^{1}$ Vanderbilt University Medical Center; ${ }^{2}$ Vanderbilt University

School of Medicine

OBJECTIVES/GOALS: We compared the validity of an International Classification of Diseases, Clinical Modification (ICD) algorithm for identifying high-grade cervical intraepithelial neoplasia and adenocarcinoma in situ (together referred to as CIN2+) from ICD 9th revision (ICD-9) and 10th revision (ICD10) codes. METHODS/STUDY POPULATION: Using Tennessee Medicaid data, we identified cervical diagnostic procedures in 2008-2017 among females aged 18-39 years in Davidson County, TN. Gold-standard cases were pathology-confirmed CIN2+ diagnoses validated by HPV-IMPACT, a population-based surveillance project in catchment areas of five US states. Procedures in the ICD transition year (2015) were excluded to account for implementation lag. We pre-grouped diagnosis and procedure codes by theme. We performed feature selection using least absolute shrinkage and selection operator (LASSO) logistic regression with 10-fold cross validation and validated models by ICD-9 era (2008-2014, $\mathrm{N}=6594)$ and ICD-10 era (2016-2017, $\mathrm{N}=1270)$. RESULTS/ ANTICIPATED RESULTS: Of 7864 cervical diagnostic procedures, 880 (11\%) were true CIN2 + cases. LASSO logistic regression selected the strongest features of case status: Having codes for a CIN2 + tissue diagnosis, non-specific CIN tissue diagnosis, high-grade squamous intraepithelial lesion, receiving a cervical treatment procedure, and receiving a cervical/vaginal biopsy. Features of non-case status were codes for a CIN1 tissue diagnosis, Pap test, and HPV DNA test. The ICD-9 vs ICD-10 algorithms predicted case status with $68 \%$ vs $63 \%$ sensitivity, $95 \%$ vs $94 \%$ specificity, $63 \%$ vs $64 \%$ positive predictive value, $96 \%$ vs $94 \%$ negative predictive value, $92 \%$ vs $89 \%$ accuracy, and C-indices of 0.95 vs 0.92 , respectively. DISCUSSION/ SIGNIFICANCE OF IMPACT: Overall, the algorithm's validity for identifying CIN2+ case status was similar between coding versions. ICD-9 had slightly better discriminative ability. Results support a prior study concluding that ICD-10 implementation has not substantially improved the quality of administrative data from ICD-9.

4489

Association between surgery with general anesthesia and cognitive decline in older adults: analysis using shared parameter models for informative dropout Phillip Schulte ${ }^{1}$, Katrina Devick ${ }^{1}$, and Juraj Sprung ${ }^{1}$

${ }^{1}$ Mayo Clinic

OBJECTIVES/GOALS: Recent studies have assessed the association between surgery with general anesthesia and cognitive decline in longitudinal cohorts of older adults. Patients diagnosed with dementia more frequently drop out of these longitudinal studies or are unable to complete the test battery. We revisit this aim with focus on methods for informative dropout. METHODS/STUDY POPULATION: We use data from the Mayo Clinic Study of Aging (MCSA), a longitudinal epidemiological study of the prevalence, incidence, and risk factors for mild cognitive impairment (MCI) and dementia. Our primary outcome of interest was global cognitive $z$-score, assessed at study visits every 15 months. We implement linear mixed effects models to assess the association between post-enrollment exposure to surgery/anesthesia and subsequent cognitive decline trajectories. Demented patients more frequently drop out of MCSA, so, subjects with the worst cognitive outcomes are unobserved and missing data may be informative. Since this missingness may be missing not at random, we use shared parameter models to analyze continuous cognitive outcomes while jointly modeling time to dementia. RESULTS/ANTICIPATED RESULTS: A total 1948 subjects, non-demented at baseline, from the MCSA were included. Median age was $79,51 \%$ of subjects were male, and $16 \%$ had MCI at enrollment. Among median follow-up of 4 study visits over median 5.4 years, 172 patients developed dementia and dropped out from further assessments of cognitive function. In adjusted linear mixed effects models, our data suggest post-enrollment exposure to surgery/anesthesia is associated with a decline in cognitive function over time (change in slope $=-0.07$ standard deviations of cognitive $z$-score per year, $95 \% \mathrm{CI}=-0.08,-0.05, \mathrm{p}<.001)$. After adjusting for informative dropout using shared parameter models, surgery/ anesthesia is associated with greater cognitive decline (change in slope $=-0.14$ per $\quad$ year, $95 \% \mathrm{CI}=-0.16, \quad-0.12, \quad \mathrm{p}<.001)$. DISCUSSION/SIGNIFICANCE OF IMPACT: We revisited a prior analysis by our group with consideration of informative dropout. Subjects who dropout due to dementia may have different trajectories of cognitive decline compared to non-demented subjects. Shared parameter models estimate the association between surgery/anesthesia and cognitive decline accounting for informative dropout.

4252

\section{Automated Fetal Brain Volumetry on Clinical Fetal MRI Using Convolutional Neural Network}

Carol Tran ${ }^{1}$, Orit Glenn ${ }^{1}$, Christopher Hess ${ }^{1}$, and Andreas

Rauschecker $^{1}$

${ }^{1}$ University Of California, San Francisco

OBJECTIVES/GOALS: We seek to develop an automated deep learning-based method for segmentation and volumetric quantification of the fetal brain on T2-weighted fetal MRIs. We will evaluate the performance of the algorithm by comparing it to gold standard manual segmentations. The method will be used to create a normative sample of brain volumes across gestational ages. METHODS/ STUDY POPULATION: We will adapt a U-Net convolutional neural network architecture for fetal brain MRIs using 3D volumes. After re-sampling $2 \mathrm{D}$ fetal brain acquisitions to $3 \mathrm{~mm}^{3} 3 \mathrm{D}$ volumes using linear interpolation, the network will be trained to perform automated brain segmentation on 40 randomly-sampled, normal fetal brain MRI scans of singleton pregnancies. Training will be performed in 3 acquisition planes (axial, coronal, sagittal). Performance will be evaluated on 10 test MRIs (in 3 acquisition planes, 30 total test samples) using Dice scores, compared to radiologists' manual segmentations. The algorithm's performance on measuring total brain volume will also be evaluated. RESULTS/ANTICIPATED RESULTS: Based on the success of prior U-net architectures for volumetric segmentation tasks in medical imaging (e.g. Duong et al., 2019), we anticipate that the convolutional neural network will accurately provide segmentations and associated volumetry of fetal brains in fractions of a second. We anticipate median Dice scores greater than 0.8 across our test sample. Once validated, the method will retrospectively generate a normative database of over 1500 fetal brain volumes 
across gestational ages (18 weeks to 30 weeks) collected at our institution. DISCUSSION/SIGNIFICANCE OF IMPACT: Quantitative estimates of brain volume, and deviations from normative data, would be a major advancement in objective clinical assessments of fetal MRI. Such data can currently only be obtained through laborious manual segmentations; automated deep learning methods have the potential to reduce the time and cost of this process.

4420

\section{Characterizing medical comorbidity prior to autism diagnosis in children before age two. \\ Michelle D Failla ${ }^{1}$, Kyle Schwartz ${ }^{1}$, Shikha Chaganti ${ }^{1}$, Tiffany G Woynaroski, PhD, CCC-SLP ${ }^{1}$, Laurie Cutting ${ }^{2}$, Bennett Landman², and Carissa Cascio ${ }^{1}$ \\ ${ }^{1}$ Vanderbilt University Medical Center; ${ }^{2}$ Vanderbilt University}

OBJECTIVES/GOALS: Autism spectrum disorder (ASD) is a developmental disorder with a high financial and personal burden. Individuals with ASD experience significant comorbid medical conditions. We identified conditions that appear prior to ASD diagnosis in order to potentially improve early screening practices. METHODS/STUDY POPULATION: We used electronic health record data from an anonymized database at Vanderbilt University Medical Center for individuals with ASD and matched controls to analyze comorbid conditions prior to an ASD diagnosis. Data were censored to include only individuals who first appeared in the databank prior to two years of age $\left(n_{\text {total }}=1551, n_{\text {controls }}=976\right.$, $\left.\mathrm{n}_{\mathrm{ASD}}=575\right)$. Comorbidities $(\sim 1800$ conditions $)$ were compared between the ASD and matched control group using a novel tool (pyPheWAS) to examine presence, count, and duration of comorbidities that occurred between 0-2 years old and before ASD diagnosis. RESULTS/ANTICIPATED RESULTS: Convulsions ( $p=0.000404, \beta=0.807)$, constipation $(p=0.000789$, $\beta=0.894)$, and strabismus $(p=0.00243, \beta=1.155)$ were the most significant comorbid conditions prior to age 2 in individuals who would later be diagnosed with ASD. The group with ASD also had more visits associated with convulsions $(p=0.00511$, $\beta=0.195)$, diseases of the esophagus $(p=0.0117, \beta=1.675)$, and allergic reactions to food $(\mathrm{p}=0.0119, \beta=0.540)$ prior to their diagnosis. The ASD group was also seen for a longer duration regarding convulsions $(p=0.000273, \beta=0.695)$, constipation $(p=0.00157$, $\beta=0.712)$, and malaise and fatigue $(p=0.00188, \beta=0.903)$ before ASD diagnosis. DISCUSSION/SIGNIFICANCE OF IMPACT: Precise comorbid condition profiles in early childhood may help uncover biomarkers leading to better prediction of a future ASD diagnosis. Medical conditions that precede the onset of measurable behavioral symptoms may enhance early screening, treatment, and intervention in ASD.

4227

\section{Closing the cross-institutional referral loop: Applying} human factors to improve consultations

April Savoy $^{1}$, Michael Weiner ${ }^{1}$, and Teresa Damush ${ }^{1}$

${ }^{1}$ Indiana University-Purdue University at Indianapolis

OBJECTIVES/GOALS: Although referrals for specialty consultations are a core clinical process, they are prone to coordination and communication breakdowns that have led to adverse clinical outcomes. This project's objective is to improve timely documentation, transmission, access, and quality of consultation notes across healthcare systems. METHODS/STUDY POPULATION: There are two specific aims for this project. In Aim 1, we will characterize clinical workflows and information flow during cross-institutional referrals. In Aim 2, we will develop and test a prototype leveraging electronic health information exchange (HIE) to increase closing the loop for cross-institutional referrals and improve the quality of consultation notes. To accomplish these aims, we will use human factors methods, including data analytics, medical-record reviews, semi-structured interviews of consultants, rapid prototyping, and usability evaluations. RESULTS/ ANTICIPATED RESULTS: Results will inform the design and integration of clinician-facing technologies into clinical workflows to close the referral loop and improve diagnostic processes. Aim 1 will provide quantitative evidence about the quality of cross-institutional referrals, inform the eventual implementation of our prototype, and identify user interface features required for successful electronic health information exchange. Based on the results from Aim 1, reports and visual representations will be generated to illustrate information flows and clinical workflows. This will prioritize design efforts for the intervention's prototype. Aim 2 will translate clinicians' requirements into prototype features and assess clinicians' experience with the prototype. DISCUSSION/SIGNIFICANCE OF IMPACT: The use and usefulness of HIE has been limited due to usability and implementation issues. Cross-institutional referrals are complex and dependent on HIE due to EHRs' lack of interoperability. This project will provide evidence-based recommendations for the use of Fast Healthcare Interoperability Resources (FHIR) to improve HIE during referrals.

4521

\section{Collecting, Interpreting and Utilizing Retrospective Clinical Data from Data Warehouses}

Brandon Joseph Sonn, PhD Candidate ${ }^{1}$, and Andrew Monte ${ }^{1}$

${ }^{1}$ University of Colorado at Denver

OBJECTIVES/GOALS: Utilizing clinical electronic health record (eHR) data pulled en masse from data warehouses provides unique challenges when applying it to retrospective studies. Use of this data in conjunction with metabolomic and genomic results to predict response to lisinopril or ondansetron has been completed. METHODS/STUDY POPULATION: Study population consists of $>2000$ subjects recruited from the Emergency Medicine Specimen Bank at University of Colorado Denver (UCD). All patients presenting to the emergency department are approached to participate which significantly increases demographic diversity of our study populations. Clinical data is pulled from Health Data Compass (data warehouse at UCD that collects all electronic health record (EHR) data to be able to deliver de-identified). Effectiveness of lisinopril and ondansetron were investigated using metabolomic data collected via ultra-high performance liquid chromatography mass spectrometry and genomic data from Illumina chip technology to find relevant correlations. RESULTS/ANTICIPATED RESULTS: Obtaining retrospective clinical data from data warehouses comes with significant challenges to be addressed. Verifying all clinical variables from patient EHRs is a crucial step that requires extensive quality control steps. As well, ensuring data validity, appropriateness of data points pulled as relate to the study criteria and identifying alternate EHR data points is needed. Chart review is a critical step necessary to surmount these challenges. Additionally, use of retrospective EHR data often necessitates the development of novel definitions of clinical effectiveness that can be abstracted from the EHRsuch as how to determine decrease in nausea without a visual analogue scale. DISCUSSION/SIGNIFICANCE OF IMPACT: Utilizing 